\title{
The effect of the type of physical activity on the perceived stress level in people with activity limitations
}

\author{
Kyo-Man Koo', Chun-Jong Kim²,* \\ 'Department of Adapted Physical Activity, Baekseok University, Cheonan, Korea \\ ${ }^{2}$ Department of Special Physical Education, Yeungnam University, Gyeongsan, Korea
}

The purpose of this study was to investigate the effect of the type of physical activity that people with activity limitations participate in on their perceived stress level. We analyzed the data of the Korean National Health and Nutrition Examination Survey (KNHANES) conducted annually from 2007 to 2015 . The subjects of the study were adults aged 19 or older who had activity limitations. The data analysis was done using the SPSS ver. 18.0 program, and complex sampling design frequencies analysis and logistic regression analysis were conducted. The statistical significance level was set at $P<0.05$. The results of this study are as follows: First, in the case of males with activity limitations, the odds ratio $(O R)$ for the perceived stress level for those who participated in walking exercise compared to those who did not was 0.707 ( $95 \%$ confidence interval [CI], 0.535-0.934; $P<0.05)$. Second, for males with activity limitations, the OR for the perceived stress level for those who participated in walking exercise for 5 days or more compared to the nonparticipation group was 0.630 ( $95 \% \mathrm{Cl}, 0.472-0.841 ; P<0.05)$. Third, for females with activity limitations, the $\mathrm{OR}$ for the perceived stress level for those who participated in flexibility exercise compared to those who did not was 0.869 (95\% Cl, 0.727-1.039; $P<0.05$ ). Fourth, for females with activity limitations, the $\mathrm{OR}$ for the perceived stress level for those who participated in flexibility exercise for 5 days or more compared to the nonparticipation group was $0.672(95 \% \mathrm{Cl}, 0.539-0.838 ; P<0.05)$.

Keywords: Physical activity, People with activity limitations, Perceived stress, Korean National Health and Nutrition Examination Survey

\section{INTRODUCTION}

People living in modern society are exposed to a lot of stress because of their need to adapt to and live in complicated and diverse environments. In particular, harmful stress causes various health problems, so it is said to be the root of all kinds of diseases. Most studies have shown that experiencing stress inhibits attempts to perform physical activity (Stults-Kolehmainen and Sinha, 2014) and causes depression (Lee and Kim, 2015). Furthermore, it is known to affect suicidal impulses in various age groups (Feskanich et al., 2002). In this way, stress has very negative effects on mental health and may thereby cause secondary problems.

Aerobic exercise has been reported to protect people from the harmful consequences of stress (Salmon, 2001), and it was also reported that when more than a certain amount of physical activity is performed during leisure time, it lowers the probability of feeling stressed (Aldana et al., 1996). Regular exercise is also closely related to stress and the improvement of stress coping abilities (Edwards, 2006), and behavioral interventions such as physical activity reduce the burden of chronic stress (McEwen, 2007). Furthermore, people who exercise 2 or 3 times a week have been reported to exhibit a lower level of stress than those who exercise less frequently or who do not exercise at all (Hassmén et al., 2000). In addition, a study which assessed mental health before and after performing aerobic exercise and weight-training for 24 weeks found significant changes in the stress level and mental health (Atlantis et al., 2004). In addition, it has been reported that the stress level decreases with increasing physical activity, such as jogging in leisure time (Schnohr et al., 2005) Furthermore, aerobic fitness training of high intensity has been shown to
${ }^{*}$ Corresponding author: Chun-Jong Kim (iD https://orcid.org/0000-0002-6715-6785 Department of Special Physical Education, Yeungnam University, 280 Daehak-ro, Gyeongsan 38541, Korea

Tel: +82-53-810-3143, Fax: +82-53-810-4671, E-mail: cjkim05@yu.ac.kr

Received: March 24, 2018 / Accepted: May 1, 2018
This is an Open Access article distributed under the terms of the Creative Commons Attribution Non-Commercial License (http://creativecommons.org/licenses/by-nc/4.0/) which permits unrestricted non-commercial use, distribution, and reproduction in any medium, provided the original work is properly cited. 
be effective in lowering the stress level (Norris et al., 1992).

As described above, appropriate physical activity has a positive effect on stress, improves mood (Arent et al., 2000), reduces dissatisfaction with life (Stults-Kolehmainen and Sinha, 2014), and improves quality of life (Atlantis et al., 2004). In addition, aerobic exercise and strength training significantly reduce depressive symptoms (Paluska and Schwenk, 2000), and a moderate level of exercise improves mood (Peluso and Guerra de Andrade, 2005). Exercise also has beneficial effects on patients taking antidepressants (Blumenthal et al., 2007). Moreover, it may change their emotional state positively (Shin, 1999). In this way, adequate types and levels of physical activity have positive effects on mental health and quality of life, as well as stress (Atlantis et al., 2004).

However, there is still a lack of research into what kind of physical activity has a positive effect on stress. In particular, people with activity limitations, such as disabled people, experience stress not only due to their physical or cognitive impairments, but also due to their negative social perception or attitudes. Therefore, people with activity limitations are relatively more likely to be exposed to harmful stress. Nevertheless, there have not been many studies on ways to manage or cope with the stress of people with activity limitations. In particular, there has been little research into the effects of physical activity, which is closely related to stress. Thus, we thought it would be very meaningful to investigate what type of physical activity affects the stress of people with activity limitations.

Therefore, the aim of this study was to investigate the effect of physical activity on stress in people with activity limitations according to the type of physical activity in which they participate, and the results of this study are expected to provide the basic data necessary to deal with the stress of people with activity limitations.

\section{MATERIALS AND METHODS}

\section{Subjects}

The purpose of this study was to investigate the effect of physical activity on the perceived stress level in participants with activity limitations according to the type of physical activity by conducting a secondary analysis using the raw data of the Korean National Health and Nutrition Examination Survey (KNHANES). The KNHANES was conducted with the approval of the Research Ethics Review Committee of the Korea Centers for Disease Control and Prevention. The data obtained from this survey are statistical data representative of the Republic of Korea obtained through an annual survey conducted every year by extracting the samples by stratified cluster systematic sampling. In this study, to increase the statistical power, the data from the 4th, 5th, and 6th periods of the years 2007-2015 were vertically combined and analyzed. The data collection for the 4th period was carried out from 2007 to 2009, with 24,871 persons surveyed in 500 enumeration districts nationwide, and the data collection for the 5 th period was conducted from 2010 to 2012, with 31,596 participants in 576 enumeration districts across the country. The data for the 6th period were collected from 2013 to 2015, and 22,948 people participated in 576 enumeration districts across the country. The data of a total of 2,397 males (estimated value, 1,535,640 persons) and 4,238 females (estimated value, 2,218,561 persons) who reported that they had activity limitations, such as orthopedic injury, heart disease, respiratory problems, stroke, diabetes, hypertension, cancer, visual or hearing impairment, emotional problems, intellectual disability, obesity, or old age, were used for the analysis. The estimated values and percentages according to the general demographic characteristics of the subjects are shown in Table 1 below.

\section{Research variables}

The research variables of this study were the type of physical activity and the perceived stress level. The variables were categorized and analyzed for the purpose of this study in accordance with the Guidelines for Using Raw Data of the KNHANES (2016). First, for physical activity, which is the independent variable, walking exercise, strength exercise, and flexibility exercise were selected as the independent variables. For walking exercise, the survey participants were asked to report 'How many days did you walk at least $10 \mathrm{~min}$ at a time in the last week?' by choosing a response among the options of 'never,' ' 1 day,' '2 days,' ' 3 days,' ' 4 days, ' ' 5 days,' ' 6 days,' and '7 days (every day).' For the purpose of this study, the responses were recategorized into ' 1 . never' and '2. I participated in it.' In the case of strength exercise, the respondents were asked to report 'How many days did you do your strength exercises such as push-ups, sit-ups, dumbbell exercises, weight lifting, and chin-ups in the last week?' by choosing a response among the options of 'never,' ' 1 day,' ' 2 days,' ' 3 days,' ' 4 days,' and ' 5 days or more.' In this study, the responses were categorized into '1. never' and '2. I participated in it.' For flexibility exercise, the respondents were asked to report 'How many days did you perform flexibility exercises such as stretching and free gymnastics in the last week? by choosing a response among the 
Table 1. Characteristics of subjects

\begin{tabular}{|c|c|c|c|c|c|c|}
\hline \multirow{2}{*}{ Characteristic } & \multicolumn{3}{|c|}{ Male } & \multicolumn{3}{|c|}{ Female } \\
\hline & Unweighted (n) & Weighted (n) & $\%$ (weighted) & Unweighted (n) & Weighted (n) & $\%$ (weighted) \\
\hline \multicolumn{7}{|l|}{ Age group (yr) } \\
\hline $19-29$ & 127 & 173,119 & 11.3 & 150 & 157,610 & 7.1 \\
\hline 30-39 & 146 & 143,722 & 9.4 & 267 & 177,291 & 8.0 \\
\hline $40-49$ & 211 & 217,946 & 14.2 & 404 & 278,083 & 12.5 \\
\hline $50-59$ & 416 & 355,623 & 23.2 & 765 & 451,456 & 20.3 \\
\hline $60-69$ & 646 & 301,688 & 19.6 & 1,157 & 496,729 & 22.4 \\
\hline 70-79 & 682 & 268,037 & 17.5 & 1,144 & 487,971 & 22.0 \\
\hline$\geq 80$ & 169 & 75,506 & 4.9 & 351 & 169,421 & 7.6 \\
\hline \multicolumn{7}{|l|}{ Household income } \\
\hline Low & 1,025 & 558,724 & 36.9 & 1,813 & 849,701 & 41.9 \\
\hline Middle-low & 645 & 418,351 & 27.6 & 1,072 & 592,335 & 26.7 \\
\hline Middle-high & 358 & 275,932 & 18.2 & 670 & 376,533 & 14.2 \\
\hline High & 335 & 261,624 & 17.3 & 569 & 334,276 & 17.3 \\
\hline \multicolumn{7}{|l|}{ Education level } \\
\hline Elementary school and below & 1,040 & 525,863 & 34.4 & 2,719 & $1,241,158$ & 56.2 \\
\hline Middle school & 406 & 246,280 & 16.1 & 450 & 251,758 & 11.4 \\
\hline High school & 601 & 470,777 & 30.8 & 703 & 480,537 & 21.8 \\
\hline College and over & 340 & 287,038 & 18.8 & 351 & 235,489 & 10.7 \\
\hline \multicolumn{7}{|l|}{ Economic activities } \\
\hline Employed & 1,080 & 732,431 & 48.0 & 1,292 & 691,095 & 31.4 \\
\hline Unemployed & 1,302 & 792,698 & 52.0 & 2,913 & $1,509,326$ & 68.6 \\
\hline
\end{tabular}

options of '1. never,' '1 day,' '2 days,' '3 days,' '4 days,' and '5 days or more.' In this study, the responses were categorized into ' 1 . never' and '2. I participated in it.'

For the perceived stress level, which is the dependent variable, the survey respondents were asked to answer the question 'How much do you feel stressed in everyday life?' by choosing a response among the options of '1. greatly,' '2. quite a lot,' '3. a little,' and '4. Hardly at all.' The raw data were recategorized into '0. a little' and '1. a lot'.

Among the independent variables, the variables that were later found to affect stress were again reanalyzed by categorizing them into 'never,' '1-2 days,' '3-4 days,' and '5 days or more,' respectively. In the process of restructuring the selected research variables, they were used for the analysis through a review of previous studies and consultation among the researchers.

\section{Data analysis}

The purpose of this study was to investigate the effect of physical activity on stress according to the type of physical activity in people with activity limitations. To achieve this goal, we employed the complex sampling design recommended in the Guidelines for Using Raw Data of the KNHANES (2016). For this purpose, the data from 2007 to 2015 were combined vertically. The variance estimation layer was assigned to the stratification variable, and the enumeration district was assigned to the cluster. For the sample weights, the integrated weights were calculated and applied to the analysis as the data sets from 2007 to 2015 were combined, and a plan file was generated before the analysis was performed. The combination ratios were $0.5 / 8.5$ for the data of the year 2007 and 1/8.5 for the data of the years 2008-2015.

A complex sampling frequencies analysis was performed to identify the characteristics of the subjects, and complex sampling logistic regression was performed to investigate the effect of each type of physical activity on stress. A variance estimation was performed after eliminating the missing cases. The user-missing values were changed to 'treat as valid' in order to prevent the occurrence of bias in the variance estimator, and the data with missing cases in the analysis variables (i.e., the variables to be analyzed) were included in the analysis (Korea Centers for Disease Control and Prevention, 2016). All statistical analyzes were analyzed using the IBM SPSS ver. 18.0 (IBM Co., Armonk, NY, USA) and the significance level was set at $P<0.05$. 
Table 2. Results of logistic regression analysis of the effect of each type of physical activity on the perceived stress level in people with activity limitations

\begin{tabular}{|c|c|c|c|c|c|c|}
\hline \multirow{2}{*}{ Parameter } & \multicolumn{3}{|c|}{ Male } & \multicolumn{3}{|c|}{ Female } \\
\hline & $\mathrm{OR}$ & $95 \% \mathrm{Cl}$ & $P$-value & $\mathrm{OR}$ & $95 \% \mathrm{Cl}$ & $P$-value \\
\hline \multicolumn{7}{|l|}{ Walking exercise } \\
\hline Nonparticipation & Reference & & & Reference & & \\
\hline Participation & 0.707 & $0.535-0.934$ & $0.015^{*}$ & 0.869 & 0.727-1.039 & 0.123 \\
\hline \multicolumn{7}{|c|}{ Muscular strength exercise } \\
\hline Nonparticipation & Reference & & & Reference & & \\
\hline Participation & 1.010 & $0.751-1.360$ & 0.946 & 0.905 & $0.694-1.181$ & 0.463 \\
\hline \multicolumn{7}{|l|}{ Flexibility exercise } \\
\hline Nonparticipation & Reference & & & Reference & & \\
\hline Participation & 0.928 & $0.716-1.201$ & 0.569 & 0.869 & $0.727-1.039$ & $0.004^{* *}$ \\
\hline
\end{tabular}

Adjusted for age, education level, household income, economic activities.

$\mathrm{OR}$, odds ratio; $\mathrm{Cl}$, confidence interval.

${ }^{*} P<0.05$. ${ }^{*} P<0.01$.

\section{RESULTS}

\section{The perceived stress level according to the type of physical activity}

Table 2 shows the results of the complex sampling logistic regression analysis of the effect of each type of physical activity on the perceived stress level in people with activity limitations.

As shown in Table 2, for the males, there was a significant association between walking exercise and the perceived stress level. However, it was found that strength training and flexibility exercise did not have any statistically significant association with the perceived stress level. In the case of the walking exercise, the OR for stress for the male group compared to the nonparticipation male group was 0.707 (95\% CI, 0.535-0.934; $P<0.05$ ), so the perceived stress level was found to be lower in the males who participated in walking exercise than in the males who did not.

In the case of the females, flexibility exercise was significantly associated with the perceived stress level. However, it was found that walking and strength training exercise did not have a statistically significant association with it. In the case of the females, the OR for the perceived stress level for those who participated in flexibility exercise compared to the nonparticipation female group was 0.869 (95\% CI, $0.727-1.039 ; P<0.05$ ), and this result indicates that the perceived stress level was lower in the women who participated in flexibility exercise compared to the women who did not.

The effect of walking exercise on the perceived stress level in males

The results of the complex sampling logistic regression analysis
Table 3. Results of logistic regression analysis of the effect of the walking exercise frequency on the perceived stress level in males with activity limitations

\begin{tabular}{lccc}
\hline \multirow{2}{*}{ Walking exercise } & \multicolumn{3}{c}{ Male } \\
\cline { 2 - 4 } & $\mathrm{OR}$ & $95 \% \mathrm{Cl}$ & $P$-value \\
\hline 25 Days & 0.630 & $0.472-0.841$ & $0.002^{*}$ \\
3-4 Days & 0.844 & $0.581-1.226$ & 0.372 \\
1-2 Days & 0.818 & $0.553-1.209$ & 0.313 \\
Nonparticipation & Reference & & \\
\hline
\end{tabular}

Adjusted for age, education level, household income, economic activities. $\mathrm{OR}$, odds ratio; $\mathrm{Cl}$, confidence interval.

${ }^{*} P<0.05$.

of the effect of the walking exercise frequency on the perceived stress level in the males with activity limitations are shown in Table 3 .

As shown in Table 3, for the males who participated in walking exercise 5 days or more, walking exercise was found to have a significant association with the perceived stress level. The OR for the perceived stress level of the males who participated in walking exercise for 5 days or more compared to the nonparticipation male group was 0.630 (95\% CI, 0.472-0.841; $P<0.05$ ), which indicated that the perceived stress level was lower in the males who participated in walking exercise for 5 days or more than in the males who did not participate at all.

\section{The effect of the flexibility exercise frequency on the perceived stress level in females}

Table 4 shows the results of the complex sampling logistic regression analysis of the effect of the flexibility exercise frequency on the perceived stress level in the females with activity limitations. 
Table 4. Results of logistic regression analysis of the effect of walking exercise frequency on the perceived stress level of females with activity limitations

\begin{tabular}{lccl}
\hline Flexibility exercise & \multicolumn{3}{c}{ Male } \\
\cline { 2 - 4 } & $\mathrm{OR}$ & $95 \% \mathrm{Cl}$ & $P$-value \\
\hline 2 Days & 0.672 & $0.539-0.838$ & $0.000^{* * *}$ \\
3-4 Days & 0.782 & $0.603-1.013$ & 0.063 \\
1-2 Days & 0.814 & $0.641-1.035$ & 0.093 \\
Nonparticipation & Reference & & \\
\hline
\end{tabular}

Adjusted for age, education level, household income, economic activities.

$\mathrm{OR}$, odds ratio; $\mathrm{Cl}$, confidence interval.

${ }^{* * *} P<0.001$.

As shown in Table 4, for the females who participated in flexibility exercise for 5 days or more, flexibility exercise was found to have a significant association with the perceived stress level. The OR for the perceived stress for the females who participated in flexibility exercise for 5 days or more compared to the nonparticipation female group was 0.672 (95\% CI, 0.539-0.838; $P<0.05$ ) and this result indicates that the perceived stress level was lower in the women who participated in flexibility exercise for 5 days or more than in the women who did not participate at all.

\section{DISCUSSION}

The aim of this study was to investigate the effect of physical activity on the perceived stress level according to the type of participation in people with activity limitations. The purpose of this study was to provide the basic data necessary to deal with the stress of people with activity limitations. For the purpose of this study, the discussion will be centered on the analyzed results.

First, for males with activity limitations, it was found that those who participate in walking exercise are less likely to feel stressed than those who do not. In addition, it was found that males who participate in walking exercise for more than 5 days are less likely to feel stressed than males who do not participate at all. These findings can be interpreted to indicate that men with activity limitations need to exercise 5 days or more a week in order to reduce their level of stress. These results were similar to those of Hassmén et al. (2000), who reported that the stress level was significantly lower in people who exercised two or more times per week. Moreover, a previous study conducted with female participants reported that walking exercise improved the emotional state, and this study result can also be interpreted as partially supporting the results of the present study (Shin, 1999). In this way, regular physical activities reduce the burden of chronic stress, improve resilience (McEwen, 2007), and lead to an increase in well-being, such as an improvement of stress-coping abilities (Edwards, 2006). Arent et al. (2000) showed that chronic exercise improved the mood of the elderly, and their study results suggest that regular walking exercise can be a very effective intervention for the conversion of harmful feelings such as stress into positive ones. Therefore, males with activity limitations, such as those with disabilities, should perform walking exercise regularly 5 days or more per week to cope with harmful stress and improve their quality of life.

However, for males with limited activity, strength and flexibility exercises did not have a significant effect on the perceived stress level. This result is not consistent with the results of Atlantis et al. (2004), who reported that weight training and aerobic exercise can improve the level of stress and quality of life. Therefore, follow-up studies need to be conducted to clarify the relationship between strength training, such as weight training, and stress.

Second, for women with activity limitations, it was found that those who participate in flexibility exercise are less likely to show a high level of perceived stress than those who do not. In addition, it was found that females who participated in flexibility exercise for 5 days or more per week were less likely to exhibit a high level of perceived stress than females who did not participate in it at all. These results suggest that for women with activity limitations, flexibility exercise is more likely to lower the perceived stress level than other types of exercise and that flexibility exercise is likely to have a considerable effect if they participate in it for 5 days or more per week. This result is thought to support the study of King et al. (2000), which suggested that regular participation in stretching and flexibility exercises may improve the quality of life. However, in the case of women with activity limitations, it was found that participation in walking or strength exercises did not have a significant association with stress. These findings are in contrast with the previous research which reported that walking exercise affected the emotional state positively in elderly women (Shin, 1999) and the previous study which showed that aerobic exercise and resistance exercise improved mood (Fox, 1999). Therefore, further in-depth research should be conducted in follow-up studies.

The results of this study suggest that physical activity generally helps to cope with harmful stress and thus contribute to health and quality of life, although there are differences in the types of physical activity that have a positive effect on stress according to gender (Asztalos et al., 2009; Penedo and Dahn, 2005). Therefore, it is necessary to make effort to encourage people with activity limitations to participate in physical activities and help them to 
deal with harmful stress, which is said to be the root of many diseases.

For males with activity limitations, walking exercise had a positive effect on the perceived stress level, and the perceived stress level was found to be lower when they participated for more than five days a week. In the case of females with activity limitations, flexibility exercise had a positive effect on the perceived stress level and the perceived stress level was found to be lower when they participated in it for 5 days or more per week. Therefore, in order for people with activity limitations to reduce their level of harmful stress, it is recommended that males should participate in walking exercise and females in flexibility exercise for 5 days or more per week.

\section{CONFLICT OF INTEREST}

No potential conflict of interest relevant to this article was reported.

\section{REFERENCES}

Aldana SG, Sutton LD, Jacobson BH, Quirk MG. Relationships between leisure time physical activity and perceived stress. Percept Mot Skills 1996;82:315-321.

Arent SM, Landers DM, Etnier JL. The effects of exercise on mood in older adults: a meta-analytic review. J Aging Phys Act 2000;8:407-430.

Asztalos M, Wijndaele K, De Bourdeaudhuij I, Philippaerts R, Matton L, Duvigneaud N, Thomis M, Duquet W, Lefevre J, Cardon G. Specific associations between types of physical activity and components of mental health. J Sci Med Sport 2009;12:468-474.

Atlantis E, Chow CM, Kirby A, Singh MF. An effective exercise-based intervention for improving mental health and quality of life measures: a randomized controlled trial. Prev Med 2004;39:424-434.

Blumenthal JA, Babyak MA, Doraiswamy PM, Watkins L, Hoffman BM, Barbour KA, Herman S, Craighead WE, Brosse AL, Waugh R, Hinderliter A, Sherwood A. Exercise and pharmacotherapy in the treatment of major depressive disorder. Psychosom Med 2007;69:587-596.

Edwards S. Physical exercise and psychological well-being. South Afr J Psychol 2006;36:357-373.

Feskanich D, Hastrup JL, Marshall JR, Colditz GA, Stampfer MJ, Willett WC, Kawachi I. Stress and suicide in the Nurses' Health Study. J Epi- demiol Community Health 2002;56:95-98.

Fox KR. The influence of physical activity on mental well-being. Public Health Nutr 1999;2(3A):411-418.

Hassmén P, Koivula N, Uutela A. Physical exercise and psychological well-being: a population study in Finland. Prev Med 2000;30:17-25.

King AC, Pruitt LA, Phillips W, Oka R, Rodenburg A, Haskell WL. Comparative effects of two physical activity programs on measured and perceived physical functioning and other health-related quality of life outcomes in older adults. J Gerontol A Biol Sci Med Sci 2000;55:M7483.

Korea Centers for Disease Control and Prevention. Guideline for using raw data of the KNHANES. Cheongju (Korea): Korea Centers for Disease Control and Prevention; 2016.

Lee KJ, Kim JI. Relating factors for depression in korean working women: secondary analysis of the Fifth Korean National Health and Nutrition Examination Survey (KNHANES V). Asian Nurs Res (Korean Soc Nurs Sci) 2015;9:265-270.

McEwen BS. Physiology and neurobiology of stress and adaptation: central role of the brain. Physiol Rev 2007;87:873-904.

Norris R, Carroll D, Cochrane R. The effects of physical activity and exercise training on psychological stress and well-being in an adolescent population. J Psychosom Res 1992;36:55-65.

Paluska SA, Schwenk TL. Physical activity and mental health: current concepts. Sports Med 2000;29:167-180.

Peluso MA, Guerra de Andrade LH. Physical activity and mental health: the association between exercise and mood. Clinics (Sao Paulo) 2005; 60:61-70.

Penedo FJ, Dahn JR. Exercise and well-being: a review of mental and physical health benefits associated with physical activity. Curr Opin Psychiatry 2005;18:189-193.

Salmon P. Effects of physical exercise on anxiety, depression, and sensitivity to stress: a unifying theory. Clin Psychol Rev 2001;21:33-61.

Schnohr P, Kristensen TS, Prescott E, Scharling H. Stress and life dissatisfaction are inversely associated with jogging and other types of physical activity in leisure time-The Copenhagen City Heart Study. Scand J Med Sci Sports 2005;15:107-112.

Shin $Y$. The effects of a walking exercise program on physical function and emotional state of elderly Korean women. Public Health Nurs 1999;16:146-154.

Stults-Kolehmainen MA, Sinha R. The effects of stress on physical activity and exercise. Sports Med 2014;44:81-121. 